\title{
EXPERIMENTS ON INTERLAYER EXCHANGE COUPLING
}

\author{
P. GRÜNBERG ${ }^{a}$, M. SCHAEFER ${ }^{a}$, K. TAKANASHI ${ }^{b}$, U. RÜCKER $^{a}$, J. NASSAR $^{a *}$ \\ AND I. MERTIG ${ }^{c}$
}

\author{
a Forschungszentrum Jülich GmbH - IFF, 52425 Jülich, Germany \\ ${ }^{b}$ Institute for Materials Research, Tohoku University, Sendai 980-77, Japan \\ 'Institut für Theoretische Physik, Technische Universität Dresden \\ 01062 Dresden, Germany
}

\begin{abstract}
The results of various experiments on interlayer exchange coupling will be reviewed. After a general discussion of the theoretical understanding we deal first with $90^{\circ}$-type coupling and show that two mechanisms proposed by Slonczewski can explain our measured data. We furthermore study the coupling of Fe films across $\mathrm{Cu}_{x} \mathrm{Au}_{1-x}$ alloys. For the composition with $x=0.5$, the coupling strength strongly increases with annealing. We believe that this is due to an order-disorder structural transformation in the $\mathrm{Cu}_{x} \mathrm{Au}_{1-x}$ alloy. Finally, we present results on epitaxially grown bilayers $\mathrm{Fe} / \mathrm{EuS}(100)$ and trilayers $\mathrm{Fe} / \mathrm{EuS} / \mathrm{Fe}(100)$. In the latter at room temperature we observe a strong decrease in the coupling strength as a function of the interlayer thickness, with a characteristic length of about $0.1 \mathrm{~nm}$. At low temperatures in the bilayer we find an antiferromagnetic coupling between Fe and the adjacent ferromagnetic EuS.
\end{abstract}

PACS numbers: 75.70.-i, 75.30.Et, 75.70.Cn

\section{Introduction}

Since the discovery of antiferromagnetic interlayer coupling in 1986 [1] numerous systems have been investigated. Generally it is found that the coupling oscillates as a function of the interlayer thickness between ferro- and antiferromagnetic type [2], and the transition zones are characterized by $90^{\circ}$-type coupling [3]. The oscillation periods are in agreement with a theory which attributes the microscopic origin of the coupling to an indirect exchange of the ferromagnetic layers via the electrons of the interlayer $[4,5]$. For the $90^{\circ}$-type coupling various mechanisms have been proposed $[6,7]$ but the situation is still unclear. It is possible that different mechanisms are superimposed. We would like to review here results of investigations of the influence of magnetic impurities in the interlayer [8].

*Permanent address: Lab. de Physique des Solides, Université Paris Sud, 91405 Orsay, France. 
We have furthermore studied the coupling across $\mathrm{Cu}_{x} \mathrm{Au}_{1-x}$ alloys [9]. The coupling strength $S$ decreases remarkably with alloying. The long period of the oscillation of the coupling decreases with increasing $x$ while the short period is almost independent of $x$. There is also an effect of annealing on $S$ which is particularly strong for the composition with $x=0.5$. With annealing, $S$ strongly increases. We believe that this is due to a order-disorder structural transformation.

Finally, we review results on epitaxially grown bilayers $\mathrm{Fe} / \mathrm{EuS}(100)[10]$ and trilayers of $\mathrm{Fe} / \mathrm{EuS} / \mathrm{Fe}(100)$. The combination of $\mathrm{Fe}$ as a transition metal and EuS as a semiconducting rare-earth ferromagnet with $T_{\mathrm{C}}=16 \mathrm{~K}$ gives the opportunity to investigate the influence of ferromagnetic order in the interlayer on the coupling strength $S$ across the interlayer. At room temperature we observe a strong decrease in $S$ as a function of the interlayer thickness, with a characteristic length of about $0.1 \mathrm{~nm}$. At low temperatures in the bilayer we find antiferromagnetic interface coupling between $\mathrm{Fe}$ and the adjacent ferromagnetic EuS.

\section{Bilinear and biquadratic coupling in $\mathrm{Fe} / \mathrm{Au} / \mathrm{Fe}$ and outline of theory}

For a quantitative evaluation of experiments the coupling is treated phenomenologically by means of an expression for the interlayer coupling areal energy density of the following kind:

$$
\begin{aligned}
E_{i}= & -J_{1} \frac{M_{1} \cdot M_{2}}{\left|M_{1}\right| \cdot\left|M_{2}\right|}-J_{2}\left(\frac{M_{1} \cdot M_{2}}{\left|M_{1}\right| \cdot\left|M_{2}\right|}\right)^{2}= \\
& -J_{1} \cos (\Delta \varphi)-J_{2}[\cos (\Delta \varphi)]^{2} .
\end{aligned}
$$

Here $\Delta \varphi$ is the angle between the magnetizations $M_{1}$ and $M_{2}$ of the films on both sides of the interlayer. $J_{1}$ and $J_{2}$ are parameters describing the kind and the strength of the coupling. If $J_{1}$ dominates then from the minima of (1) the coupling is ferromagnetic (F) (antiferromagnetic (AF)) for positive (negative) $J_{1}$ respectively. In the same way if $J_{2}$ dominates and is negative, the minima of (1) correspond to $90^{\circ}$-coupling. The first term on the left hand side of Eq. (1) is often called bilinear- and the second biquadratic coupling. The parameters $J_{1}$ and $J_{2}$ can be determined from measured $M(H)$ curves and from the frequencies of coupled spin-wave modes.

An important milestone in the exploration of the interlayer coupling phenomena was the discovery that the parameters $J_{1}$ and $J_{2}$ display damped oscillations which can be multiperiodic, i.e. different oscillation periods can be superimposed. This was first found for the $\mathrm{Fe} / \mathrm{Cr}-[11,12]$ and for the $\mathrm{Fe} / \mathrm{Au}$-system [13] and later on for many others. The result for the $\mathrm{Fe} / \mathrm{Au}$-system is displayed in the main part of Fig. 1. There are clearly two superimposed oscillation periods $\lambda_{1}, \lambda_{2}$.

At the same time theories were developed which explain interlayer exchange coupling in terms of an indirect coupling of the magnetic films via the metal electrons in the interlayer. There is a strong similarity to the famous Ruderman-KittelKasuya-Yoshida (RKKY) interaction which describes the interaction of isolated magnetic moments in metallic host lattices and in rare-earth metals.

One important result of these theories was the discovery that the different oscillation periods are associated with certain extremal distances - also called 


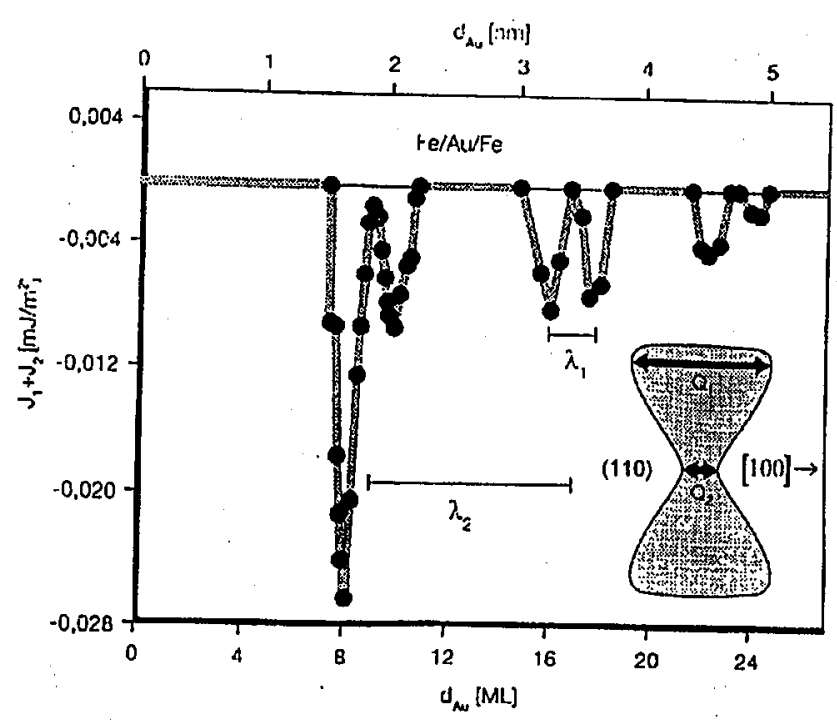

Fig. 1. Interlayer coupling of $\mathrm{Fe}$ layers across $\mathrm{Au}$ interlayers as a function of the $\mathrm{Au}$ thickness. Only the part with negative parameter $J_{1}+J_{2}$ is shown, due to the fact that these data were obtained without "spin engineering". The insert shows a cross-section of the Fermi surface of Au parallel to a (110)-plane. The two distances $Q_{1}$ and $Q_{2}$ in the "dog's bone orbit" in the [100]-direction are connected with the two oscillation periods $\lambda_{1}$ and $\lambda_{2}$ (see text).

calipers - in the Fermi surface of the interlayer material [4]. We concentrate here on the case where the layer normal is parallel to a [100]-direction of the Au lattice. It turns out that in this case the relevant calipers are the two extremal diameters $Q_{1}$ and $Q_{2}$ of the "dog's bone orbit" in the Fermi surface of the fcc noble metals as shown in the insert of Fig. 1. Theory then predicts that in this case oscillatory coupling should display two periods $\lambda_{1}$ and $\lambda_{2}$ which are obtained from $Q_{1}$ and $Q_{2}$ via $\lambda_{i}=2 \pi / Q_{i}(i=1,2)$. If the layer normal is [100] these are the only two relevant calipers. From this we conclude that the two periods observed in Fig. 1 are connected with the two calipers $Q_{1}$ and $Q_{2}$. From recent calculations [14] of the Fermi surface of $\mathrm{Au}$ we get $\lambda_{1}$ (theory) $=9.8 \mathrm{ML}, \lambda_{2}$ (theory) $=2.6 \mathrm{ML}$, while the experimental values from Fig. 1 are $\lambda_{1}$ (exp.) $=8.00 \mathrm{ML}, \lambda_{2}(\exp )=.2.00 \mathrm{ML}$. Hence there is also good quantitative agreement.

Although it is now rather certain that the reason for oscillatory coupling is an RKKY type mechanism, the origin of $90^{\circ}$-type coupling is not so clear. Like in the first observation [3] it seems to appear predominantly in the transition zones between $\mathrm{F}$ - and AF-type coupling. More precise analysis however reveals that this is mostly due to the fact that in these ranges the $J_{1}$-parameter changes sign and therefore is small. Hence $J_{2}$, responsible for $90^{\circ}$-type coupling, dominates. Closer examination of $J_{2}$ shows that opposite to $J_{1}$ it is more or less constant with a slight decrease when the interlayer thickness increases but shows a strong attenuation for increasing sample temperature. 


\section{Experimental studies of mechanisms for biquadratic coupling in $\mathrm{Fe} / \mathrm{Ag}(\mathrm{Fe}) / \mathrm{Fe}$}

Various mechanisms for the explanation of biquadratic coupling $J_{2}$ have been proposed. The first was the "roughness model" by Slonczewski [6]. It explains $J_{2}$ in terms of a competition effect between ferro- and antiferromagnetic type coupling (positive and negative $J_{1}$ ) caused by interface roughness. Since in many cases the temperature dependence of $J_{2}$ is stronger than expected from the roughness model, Slonczewski came up with still another idea, namely that magnetic impurities dissolved in the interlayer and their fluctuation could be responsible for $J_{2}$ [7]. The advantage of this mechanism is that it can be tested experimentally. To this end we have doped $\mathrm{Fe} / \mathrm{Ag} / \mathrm{Fe}$ structures in the midplane of the $\mathrm{Ag}$ film with various amounts of $\mathrm{Fe}$ and evaluated the coupling parameters $J_{1}$ and $J_{2}$ from their $M(H)$ loops. The amount of $\mathrm{Fe}$ dopant is given in terms of the nominal thickness $t_{\mathrm{Fe}}$, obtained with a quartz crystal monitor. Here only the fractions $J_{i}^{1 \mathrm{~s}}=J_{i}^{\text {expt }}-J_{i}^{\text {pure }}(i=1,2)$ are considered as due to the loose spins, where $J_{i}^{\text {expt }}$ are the measured values. $J_{1}^{\text {pure }}$ is the intrinsic coupling and $J_{2}^{\text {pure }}$ is thought to be due to Slonczewski's roughness mechanism. The $J_{i}^{\text {ls }}$ as a function of $t_{\mathrm{Fe}}$ are plotted in Fig. 2. For small $t_{\mathrm{Fe}} J_{1}^{\text {ls }}$ decreases linearly while $J_{2}^{\text {ls }}$ increases linearly with increasing $\mathrm{Fe}$ concentration. This linear increase is expected from the loose spin theory.

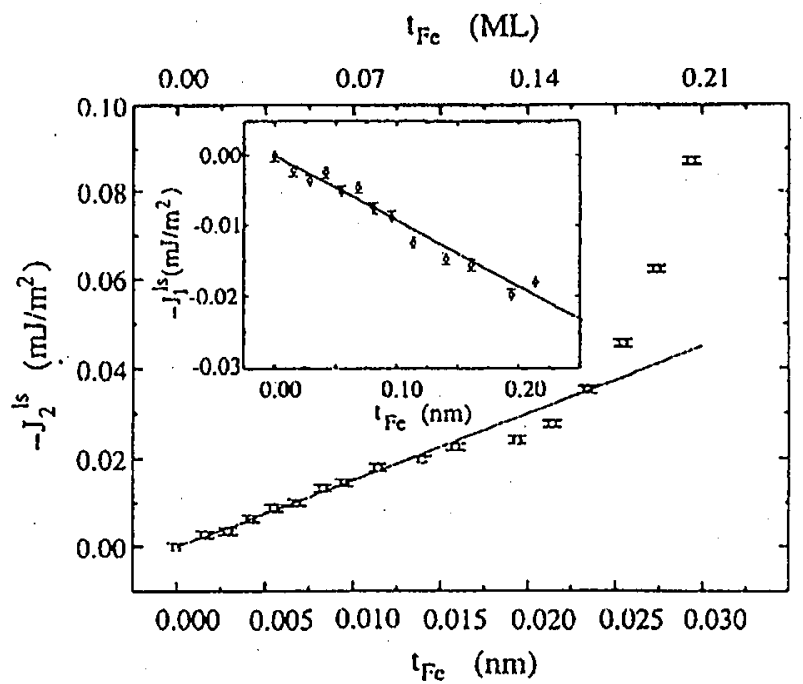

Fig. 2. $\quad J_{2}^{\mathrm{ls}}=J_{2}^{\text {expt }}-J_{2}^{\text {pure }}$ as a function of the Fe concentration in the midplane of the $\mathrm{Ag}$ interlayer, measured at $T=7 \mathrm{~K} .-J_{2}^{\mathrm{ls}}$, representing $90^{\circ}$ type coupling, increases linearly as a function of the $\mathrm{Fe}$ concentration, here given in terms of the nominal thickness as measured with the quartz crystal monitor. The solid line is a fit using the loose spin model. The inset shows $-J_{1}^{\mathrm{ls}}$, representing antiferromagnetic type coupling, as a function of $t_{\mathrm{Fe}}$, which decreases linearly with $t_{\mathrm{Fe}}$. 
The deviation from the linear dependence of $J_{2}^{\mathrm{ls}}$ on $t_{\mathrm{Fe}}$ for $t_{\mathrm{Fe}}>0.15 \mathrm{ML}$ is believed to be connected with the creation of finite size clusters of the $\mathrm{Fe}$ in the midplane of the Ag interlayer. Such superparamagnetic clusters have higher susceptibility per atom than isolated atoms and, therefore, have a different contribution to the coupling. The deflection of the experimental curve from the linear law reflects the growth of the average number of atoms in clusters with increasing Fe concentration. Generally speaking, to stay within the limits of the model, the Fe concentration $\rho$ has to be small. If $\rho$ exceeds a certain value, the spins will not be loose. Taking small concentrations considerably improved our results. This is in agreement with Heinrich et al. [15] even though they chose concentrations which in our view appear generally to be too high: Slonczewski restricted his fits to Fe concentrations $t_{\mathrm{Fe}}<0.06 \mathrm{ML}$, while Heinrich et al. worked generally with $t_{\mathrm{Fe}}<0.12 \mathrm{ML}$.

\section{Coupling across disordered and ordered alloys of $\mathrm{Cu}_{x} \mathrm{Au}_{1-x}$}

The coupling of $\mathrm{Fe}$ across $\mathrm{Au}$ is shown in Fig. 1. We further doped the $\mathrm{Au}$ interlayer with $\mathrm{Cu}$ to study also the coupling across $\mathrm{Cu}_{x} \mathrm{Au}_{1-x}$ alloys [9]. In addition, the effect of the order-disorder transformation on the coupling is investigated since the $\mathrm{Cu}_{x} \mathrm{Au}_{1-x}$ alloys have ordered structures around $x=0.25$, 0.50 and 0.75 in the equilibrium phase diagram.

$\mathrm{Fe}(1.4 \mathrm{~nm}) / \mathrm{Cu}_{x} \mathrm{Au}_{1-x} / \mathrm{Fe}(1.4 \mathrm{~nm})$ sandwich films with wedge-shaped interlayers ranging in thickness from 0 to $5-6 \mathrm{~nm}$ were prepared by the MBE technique on $\mathrm{GaAs}(100)$ substrates with thick $\mathrm{Ag}$ buffers. For the $\mathrm{Cu}_{x} \mathrm{Au}_{1-x}$ alloy formation, $\mathrm{Cu}$ and $\mathrm{Au}$ were coevaporated from independent sources on the substrates at room temperature (RT) or $80^{\circ} \mathrm{C}$. The deposition rate for each element was in the range of $0-0.05 \mathrm{~nm} / \mathrm{s}$. The compositions of the alloys were controlled by adjusting the deposition rates, and they were examined by in situ Auger electron spectroscopy. We observed quite good oscillations of the reflection high-energy electron diffraction (RHEED) intensity for the $\mathrm{Cu}_{x} \mathrm{Au}_{1-x}$ growth. The decay of the oscillation with increasing layer number was more remarkable for larger $x$.

The strength of the interlayer coupling was determined from $M(H)$ curves measured at RT through the use of the magnetooptical Kerr effect. The sum of the bilinear and biquadratic coupling constants, $J_{1}+J_{2}$, were derived from the saturation field $H_{\mathrm{s}}$ of the $M(H)$ curve.

Figure 3 shows the results of $J_{1}+J_{2}$ as a function of the thickness of the $\mathrm{Cu}_{x} \mathrm{Au}_{1-x}$ alloy with $x \approx 0.5$ after annealing. Without annealing no oscillatory coupling was observed but after annealing two oscillation periods occur similar as in Fig. 1 . In the as-deposited state, $\mathrm{Cu}_{x} \mathrm{Au}_{1-x}$ alloys prepared by coevaporation are disordered alloys. We believe that the high probability of electron scattering due to structural disorder leads to the lifetime broadening of Fermi surfaces, which diminishes the coupling strength to the point where it is unobservable. By annealing we obtain the ordered phase and the coupling appears. The self-consistent fully relativistic Korringa-Kolnn-Rostoker coherent potential approximation (KKR-CPA) calculations of the electronic structures for disordered $\mathrm{Cu}_{x} \mathrm{Au}_{1-x}$ alloys [14] indicate that the diameter of the Fermi surface is almost invariable with $x$, however, $Q_{1}$ increase considerably with increasing $x$ due to the change in the lattice para- 


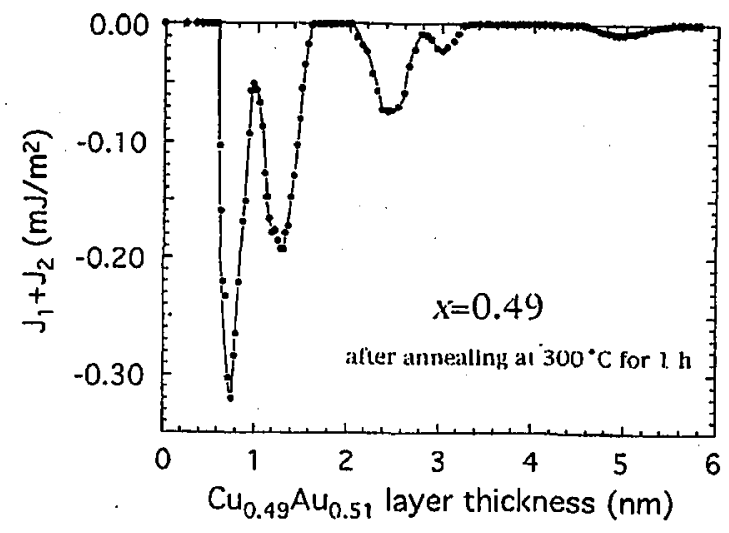

Fig. 3. Coupling strength $J_{1}+J_{2}$ of $\mathrm{Fe}$ across $\mathrm{Cu}_{0.49} \mathrm{Au}_{0.51}$ alloy after annealing at $300^{\circ} \mathrm{C}$ for $1 \mathrm{~h}$ as a function of the interlayer thickness.

meter. On the other hand, $Q_{2}$ increases slightly with increasing $x$, and the increase is negligible compared with that in $Q_{1}$. Comparison of Fig. 1 and Fig. 3 shows that the experiment is in qualitative agreement with the calculation.

\section{Coupling between Fe and EuS and of Fe across EuS}

We discuss first the observation of antiferromagnetic interlayer coupling of the ferromagnetic layers Fe and EuS which are in direct contact. The coupling is antiferromagnetic and its strength can therefore be measured from ferromagnetic saturation in an external field. The coupling strength $J$ is about $-0.2 \mathrm{~mJ} / \mathrm{m}^{2}$ at $5 \mathrm{~K}$, it decreases when the temperature increases and vanishes near the Curie temperature $\left(T_{\mathrm{C}}\right)$ of the EuS layer [10].

Samples consisting of $\mathrm{Fe}(100)$ and $\mathrm{EuS}(100)$ grown on GaAs single crystals were studied. Details about the growth of $\operatorname{EuS}(100)$ on $\mathrm{Fe}(100)$ and transport properties of the layers will be published elsewhere [16]. The pair of Fe and EuS was chosen because they both have cubic structures, the lattice mismatch in the (100) plane being only $4 \%$. This makes it possible to accomplish an epitaxial growth. Moreover, due to low $T_{\mathrm{C}}$ (about $23 \mathrm{~K}$ for our films) EuS films permit easily to change their magnetizations.

To obtain the strength of the antiferromagnetic coupling between the $\mathrm{Fe}$ - and the EuS layer, hysteresis loops were recorded. At low temperatures and low fields the magnetization of the EuS layer is oriented against the magnetic field while the magnetization of the Fe layer is parallel to it. If the field is increased to more than $80 \mathrm{mT}$ one observes the reorientation of the magnetization of the EuS layer, and for higher fields both layers are magnetized in field direction. From the value of the transition field $H_{\mathbf{t}}$ and the measured value of the $\mathrm{EuS}$ magnetization one can obtain the strength $J$ of the interlayer coupling as a function of the temperature by calculating the coupling energy per surface unit which is defined as $E_{\mathrm{c}}=$ $-J M_{\mathrm{Fe}} \cdot M_{\mathrm{EuS}} /\left|M_{\mathrm{Fe}}\right|\left|M_{\mathrm{EuS}}\right|$. The same measurements were also performed for samples with dominating $\mathrm{EuS}$ magnetization. Since in that case the reorientation of the Fe layer takes place, $H_{\mathfrak{t}}$ is different. However, both measurements, of course, 
should provide the same $J$. The result was a coupling strength of $0.2 \mathrm{~mJ} / \mathrm{m}^{2}$ at $5 \mathrm{~K}$ which vanishes near $T_{\mathrm{C}}$ of EuS. As one should expect from the Heisenberg model, the so-defined $J(T)$ and the magnetization of the EuS layer are nearly proportional to each other, although below. $10 \mathrm{~K}$ a discrepancy is clearly visible.

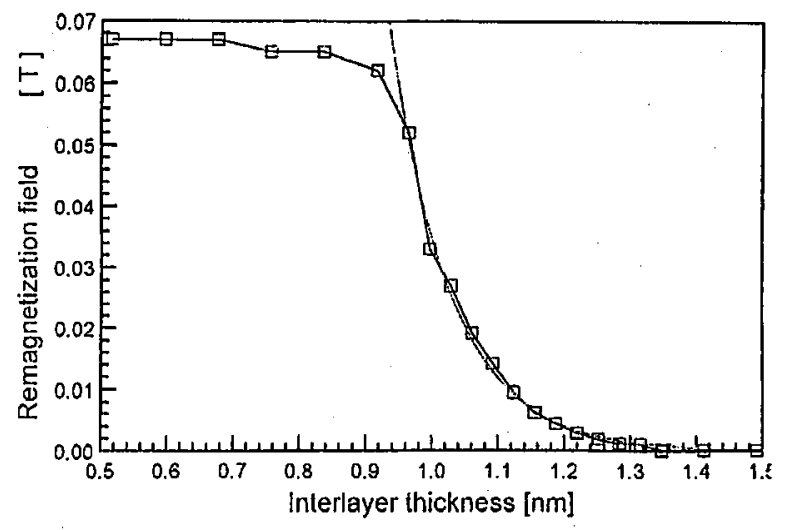

Fig. 4. Magnetic field which is needed to reverse the orientation of the magnetization of the top Fe layer, as a function of the EuS interlayer thickness in the layered system $12 \mathrm{~nm} \mathrm{Fe} / 0.83 \mathrm{~nm} \mathrm{Cr} / 3 \mathrm{~nm} \mathrm{Fe} /$ EuS-wedge/3 nm Fe.

From remagnetization curves also the coupling of Fe films across EuS interlayers can be deduced. However, since only ferromagnetic type coupling is observed, a method called "spin engineering" has to be employed. Figure 4 displays our result. The coupling strength is shown in terms of the remagnetization field of the spin-engineered samples. For interlayer thicknesses above $0.95 \mathrm{~nm}$ a remagnetization field of $0.01 \mathrm{~T}$ corresponds to a ferromagnetic interlayer coupling strength of $0.05 \mathrm{~mJ} / \mathrm{m}^{2}$. Below $0.95 \mathrm{~nm}$ the ferromagnetic coupling strength becomes too strong to be measured, due to the fact that it overrides the antiferromagnetic coupling of the spin-engineered sample. Part of this coupling might be due to $\mathrm{Fe}$ bridges (pinhole coupling) in the interlayer which might be taken into account by shifting the curve of Fig. 4 to the left in order to obtain the curve representing the coupling across the EuS interlayer material. As is indicated by the dotted line, the measured curve can be well approximated by an exponential decay with a decay length of $0.1 \mathrm{~nm}$.

\section{Concluding remarks}

Following its discovery the phenomenon of oscillatory interlayer exchange coupling has mainly been investigated in order to understand in detail its microscopic origin. Now that this has been achieved the question arises where to go further. Here we have presented a couple of examples which show that the coupling properties are linked to the structural and magnetic properties of the interlayer material and can be used to characterize them. In the same way the coupling can also be modified and tailored. The examples shown here give a flavour of what 
happens by doping the interlayers with magnetic impurities, at disorder to order structural phase transitions and at paramagnetic to ferromagnetic phase transitions.

\section{References}

[1] P. Grünberg, R. Schreiber, Y. Pang, M.B. Brodsky, H. Sowers, Phys. Rev. Lett. 57, 2442 (1986).

[2] S.S.P. Parkin, N. More, K.P. Roche, Phys. Rev. Lett. 64, 2304 (1990).

[3] M. Rührig, R. Schäfer, A. Hubert, R. Mosler, J.A. Wolf, S. Demokritov, P. Grünberg, Phys. Status Solidi A 125, 635 (1991).

[4] P. Bruno, C. Chappert, Phys. Rev. Lett. 67, 1602 (1991).

[5] D.M. Edwards, J. Mathon, R.B. Muniz, M. Villeret, J.M.Ward, in: Magnetism and Structure in Systems of Reduced Dimension, Eds. R.F.C. Farrow, B. Dieny, M. Donath, A. Fert, B.D. Hermsmeier, NATO ASI Series B: Physics, Vol. 309, Plenum Press, New York 1993, p. 401.

[6] J.C. Slonczewski, Phys. Rev. Lett. 67, 3172 (1991).

[7] J.C. Slonczewski, J. Appl. Phys. 73, 5957 (1993).

[8] M. Schäfer, S. Demokritov, S. Müller-Pfeiffer, R. Schäfer, M. Schneider, P. Grünberg, W. Zinn, J. Appl. Phys. 77, 6432 (1995).

[9] K. Takanashi, R. Schreiber, I. Mertig, P. Grünberg, J. Magn. Magn. Mater. 156, 237 (1996).

[10] U. Rücker, S. Demokritov, R.R. Arons, P. Grünberg, J. Magn. Magn. Mater. 156, 269 (1996).

[11] J. Unguris, R.J. Celotta, D.T. Pierce, Phys. Rev. Lett. 67, 140 (1991).

[12] S.T. Purcell , W. Folkerts, M.T. Johnson, N.W.E. McGee, K. Jager, J. de Stegge, W.B. Zeper, W. Hoving, P. Grünberg, Phys. Rev. Lett. 67, 903 (1991).

[13] A. Fuss, S. Demokritov, P. Grünberg, W. Zinn, J. Magn. Magn. Mater. 103, L221 (1992); A. Fuss, J.A. Wolf, P. Grünberg, Phys. Scr. Vol. T 45, 995 (1992).

[14] I. Mertig, unpublished.

[15] B.- Heinrich, J.F. Cochran, in: Ultrathin Magnetic Films, Eds. B. Heinrich, J.A.C. Bland, Vol. 1, Springer, Berlin 1994, and B. Heinrich, M. From, J.F. Cochran, M. Kowalewski, D. Atlan, Z. Celinski, K. Myrtle, J. Magn. Magn. Mater. 140-144, 545 (1995).

[16] J. Nassar, to be published. 